\title{
A practical MRI-based reconstruction method for a new endocavitary and interstitial gynaecological template
}

\author{
Jose Richart, MSc', Antonio Otal, MSc', Silvia Rodriguez, MD!, Ana Isabel Nicolás, MD², Marina DePiaggio, MD!, \\ Manuel Santos, MD', Javier Vijande, PhD 3,4, Facundo Ballester, PhD³, Jose Perez-Calatayud, PhD1,5 \\ 'Radiotherapy Department, Benidorm Hospital, Benidorm, Alicante, ${ }^{2}$ Radiology Department, Benidorm Hospital, Benidorm, Alicante, \\ ${ }^{3}$ Atomic, Molecular and Nuclear Physics Department, University of Valencia, Burjassot, Valencia, ${ }^{4}$ IFIC (CSIC-UV), Burjassot, Valencia. \\ ${ }^{5}$ Radiotherapy Department, La Fe University and Polytechnic Hospital, Valencia, Spain
}

\begin{abstract}
Purpose: There are perineal templates for interstitial implants such as MUPIT and Syed applicators. Their limitations are the intracavitary component deficit and the necessity to use computed tomography (CT) for treatment planning since both applicators are non-magnetic resonance imaging (MRI) compatibles. To overcome these problems, a new template named Template Benidorm (TB) has been recently developed. Titanium needles are usually reconstructed based on their own artifacts, mainly in T1-weighted sequence, using the void on the tip as the needle tip position. Nevertheless, patient tissues surrounding the needles present heterogeneities that complicate the accurate identification of these artifact patterns. The purpose of this work is to improve the titanium needle reconstruction uncertainty for the TB case using a simple method based on the free needle lengths and typical MRI pellets markers.

Material and methods: The proposed procedure consists on the inclusion of three small A-vitamin pellets (hyperintense on MRI images) compressed by both applicator plates defining the central plane of the plate's arrangement. The needles used are typically $20 \mathrm{~cm}$ in length. For each needle, two points are selected defining the straight line. From such line and the plane equations, the intersection can be obtained, and using the free length (knowing the offset distance), the coordinates of the needle tip can be obtained. The method is applied in both T1W and T2W acquisition sequences. To evaluate the inter-observer variation of the method, three implants of T1W and another three of T2W have been reconstructed by two different medical physicists with experience on these reconstructions.

Results and conclusions: The differences observed in the positioning were significantly smaller than $1 \mathrm{~mm}$ in all cases. The presented algorithm also allows the use of only T2W sequence either for contouring or reconstruction purposes.
\end{abstract} The proposed method is robust and independent of the visibility of the artifact at the tip of the needle.

J Contemp Brachytherapy 2015; 7, 5: 407-414 DOI: $10.5114 /$ jcb.2015.55340

Key words: brachytherapy template, catheter reconstruction, gynecological template, interstitial implants.

\section{Purpose}

In recent years, 3D image guided treatment planning has been introduced into clinical practice in gynecologic brachytherapy procedures with the goal of achieving dose conformation to the target volume and avoiding high dose areas in the organs at risk involved. Many authors have advocated the use of magnetic resonance imaging (MRI) as the preferred image modality [1, 2]. Treatment planning based exclusively on MRI is preferred to other image modalities traditionally employed, like computed tomography (CT) or methods combining both MRI and CT. Many arguments support brachytherapy based exclusively on MRI: superior soft tissue contrast, lower in- accuracies derived from registration procedures, sparing of CT-imaging doses, the fact that dose calculation algorithms do not take into account $\mathrm{CT}$ numbers because all medium is assumed water, hence saving time and increasing patient comfort; etc. The American Brachytherapy Society (ABS) and the Groupe Européen de Curiethérapie of the European Society for Radiotherapy and Oncology (GEC-ESTRO) have published recommendations about the use of MRI as the preferred image modality for imageguided brachytherapy (IGBT) [3, 4, 5].

MRI-guided BT reveals the limitations of traditional intracavitary applicators. These limitations have encouraged the development of new applicators with interstitial components added to the basic intracavitary applicator, 
using either metallic or plastic needles compatible with MRI. Some of the most widely used intracavitary applicators are based either on classic ring, named the Vienna applicator [6], or on ovoids, as the Utrecht applicator [7] (Vienna or Utrecht applicators, Nucletron, an Elekta company, Elekta AB, Stockholm, Sweden). However, these applicators, even with an additional interstitial component, present limitations in covering the lesion when extension to parametrium exists. This is the case of patients with locally advanced cervical carcinomas with bulky parametrial extension (medial or distal), bulky primary disease or disease with poor response to external beam radiation therapy (EBRT), extensive paravaginal involvement extending to middle and lower third of the vagina, or disease that has invaded the bladder or rectum (stage IVA). In such cases, interstitial approaches based on templates such as the Martinez Universal Perineal Interstitial Template (MUPIT, Martinez Universal Perineal Interstitial Template) [8] or the Syed applicator (Nucletron, an Elekta company, Elekta AB, Stockholm, Sweden) [9] have been recommended $[10,11]$.

These templates present two main issues. The first, the lack of an intracavitary component, and the second the need to use CT-based treatment planning since both templates are non-MRI compatible. To overcome these disadvantages, and based on the experience at our institution with the MUPIT, a new template named Template Benidorm (TB) has been developed. This new template implements both titanium needles and intracavitary component. Therefore, it is fully MR compatible [12].

Catheter reconstruction based only on MRI is a challenge. Some markers may be used in order to visualize the source path in some endocavitary applicators. Many authors report the use of catheters filled with $\mathrm{CuSO}_{4}$ [13], saline water [14], or glycerine [15] for this purpose. However, the use of markers for the interstitial component with titanium needles is troublesome due to its small diameter and because they do induce susceptibility artefacts, particularly at the needle tip. GEC-ESTRO provides recommendations for commissioning and applicator reconstruction in 3D image-based treatment planning of cervix cancer brachytherapy [16].

Even though applicator reconstruction can be made in $\mathrm{T} 2 \mathrm{~W}$ sequences (especially if the reconstruction is based on a library of applicators implemented in the treatment planning system), a second set of images with better visualization of source channels may be used with T1W sequences, particularly if titanium applicators or needles are present $[16,17]$. Many authors have reported the use of these kind of sequences $[13,18]$ and the use of the susceptibility artefact produced by the titanium for reconstruction purposes. However, in clinical practice, recognition of the void at the end of the needle is not always straightforward, since heterogeneities are present in tissues. That makes difficult to accurate determine the needle tip end in clinical conditions.

Hence, the purpose of this work is to present a new method to reconstruct brachytherapy interstitial implants where a large number of titanium needles are present (typical number of needles ranges between 16 and 20). Although we have used the perineal Template Benidorm
[12], the method is easily extensible to other kind of templates. In addition, the tip location of the needles obtained with this method is performed when the reconstruction is based on 3D T1W and T2W sequences.

Furthermore, an inter-observer study has been performed. Two medical physicists did the reconstruction independently and then mean differences were measured.

\section{Material and methods \\ Description of the applicator}

Rodriguez et al. have described the template Benidorm elsewhere in the literature [12]. Briefly, the template consists in two perineal plates with two central holes allowing placement of a vaginal cylinder available in different sizes to accommodate different vaginal lengths. These cylinders can also accommodate different intrauterine tubes with varying angles and lengths from Nucletron (Nucletron, an Elekta company, Elekta AB, Stockholm, Sweden). Additionally, the plates are drilled with 12 rows of holes $1.1 \mathrm{~cm}$ apart to introduce straight and angled "Trocar Point" titanium needles $1.9 \mathrm{~mm}$ in diameter and $200 \mathrm{~mm}$ length provided by Nucletron.

The plates have three dimples where three A-vitamin pellets are placed. These pellets produce a high signal in both, T1W and T2W sequences, and are used in the reconstruction process (Figure 1). The number, location, and depth of the needles are decided by the physician and customized for each patient.

\section{Image acquisition}

The MRI scans of the patients were acquired with a 1.5 T MR imager (Optima MR 450w, software version DV24, GE Medical Systems Milwaukee, Wisconsin, USA). The patients were positioned with their arms in vertical position and free breathing. An eight-channel phased array receiver coil was used according to standard clinical MR protocols.

Two sets of cases were analyzed in this study. In the first set, the acquisition consisted of two types of sequences. The first sequence used for contouring approaches GEC-ESTRO recommendations. It consists in an axial T2 weighted fast recovery fast spin echo (FRFSE) sequence. A second type of sequence is acquired to enhance contrast between titanium needles and surrounding soft tissue. This sequence is used in the reconstruction process. It was a 3D T1-weighted fast radio frequency spoiled gradient recalled echo sequence, with frequency-selective fat-suppression for catheter reconstruction. In the second set of patients, the acquisition consists of only one type of sequence axial T2W FRFSE like the one previously mentioned but with a slice thickness reduced to $2 \mathrm{~mm}$. This sequence used for both delineating and reconstruction. Table 1 summarizes some parameters of these MR sequences.

\section{Applicator reconstruction}

Clinical MR examinations were reconstructed for 16 patients of group 1 (3D T1W) and for 10 patients corresponding to group 2 (T2W). The reconstruction process 

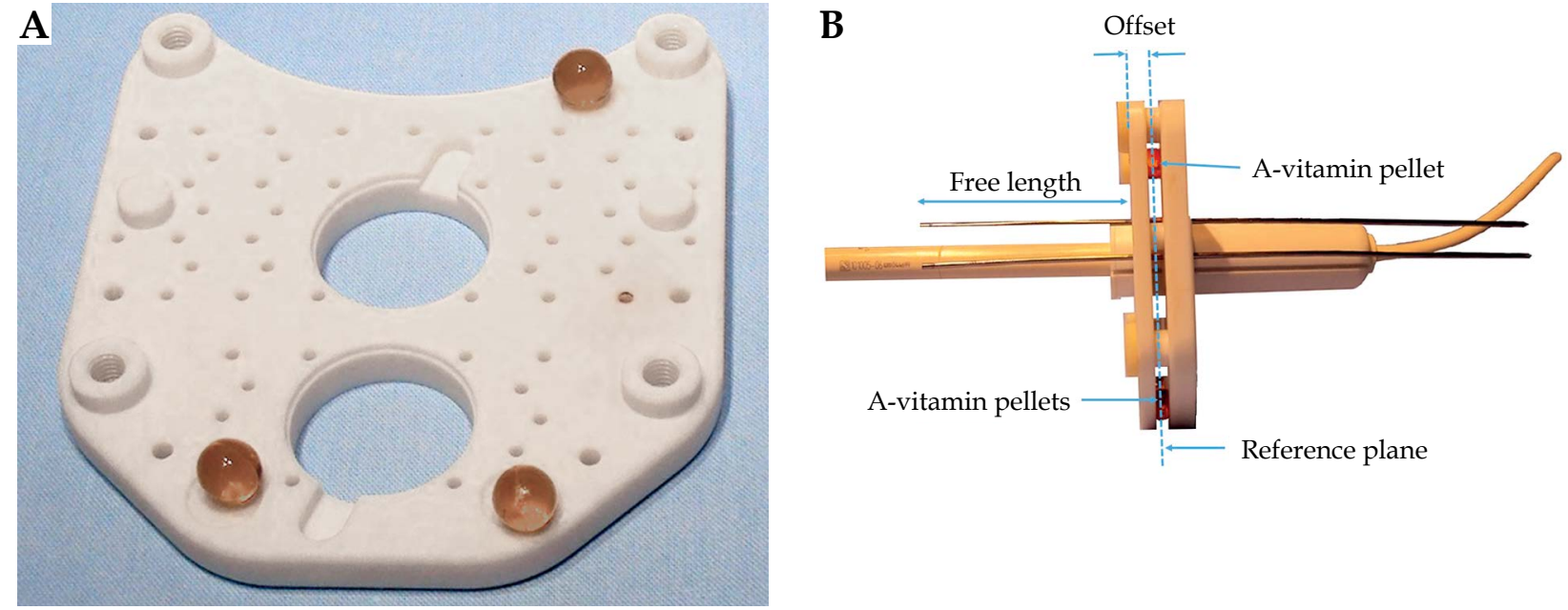

Fig. 1. A) One of the plates of the Template Benidorm with the three A-vitamin pellets in place. B) Applicator arrangement showing the vaginal cylinder, intrauterine tandem, a sample of two titanium needles, and the A-vitamin pellets in between the two perineal plates. The free length distance used in the reconstruction process is also shown

and planning was performed in the Oncentra Master Plan Treatment Planning System v4.3 from Nucletron (Nucletron, an Elekta company, Elekta AB, Stockholm, Sweden). In the cases where an intrauterine tandem was present, it was reconstructed with the help of the MR marker [14]. For the needles, the proposed reconstruction method is based on the free length measured outside of the external template plate (Figure 1B) and the position of the A-vitamin pellets as follows.

The first step consists in the location of the centers of the three pellets on MR images. Since A-vitamin appears as a hyper-intense signal on a MRI, these pellets are easily identified on the acquisition study allowing us to obtain the coordinates of their centers, B1, B2, and B3. Figure 2 shows paraxial views where the A-vitamin pellets are clearly visible both in T1W and T2W.

The coordinates of these three points B1, B2, and B3, define the reference plane shown in Figure 1B. Addition- ally, two points describe the direction for each needle, which is a straight line. These points can be anywhere on the needle, so they are chosen by selecting two separate slices that present good image contrast. Figure 3A shows an example of a case reconstructed based on 3D T1W sequence. On the left-hand side, two para-axial images show the positions of points P1 and P2. The location of these two paraxial images are represented with a green line on the upper right (sagittal) and on the lower right (coronal) frames of Figure 3A. In the same way, an example of a case reconstructed based on T2W sequence is shown in Figure 3B.

The intersection of the line defined by P1 and P2 with the aforementioned reference plane, defines a point and, together with the free length of the needle, offset distance (7 $\mathrm{mm})$, total length of the needle $(200 \mathrm{~mm})$, and some simple algebra, the coordinates of the needle tip are obtained (see Figure 4).

Table 1. Magnetic resonance imaging (MRI) acquisition parameters

\begin{tabular}{lccc}
\hline & & MR sequence set 1 & MR sequence set 2 \\
\cline { 2 - 4 } & 3D T1W & Axial T2W & Axial T2W \\
\hline Direction & 3D & Axial & Axial \\
\hline Echo time (ms) & Minimum & 105 & 68 \\
\hline Repetition time (ms) & 3.4 & 4553 & 6100 \\
\hline Echo train length & - & 15 & 19 \\
\hline Number of excitations & 3 & 4 & 4 \\
\hline Matrix size & $256 \times 224$ & $360 \times 256$ & $256 \times 192$ \\
\hline Section thickness $(\mathrm{mm})$ & 1.6 & 4 & 2 \\
\hline Increment $(\mathrm{mm})$ & 0 & 0 & 27 \\
\hline Field-of-view $(\mathrm{cm})$ & 24 & 31 & 25
\end{tabular}



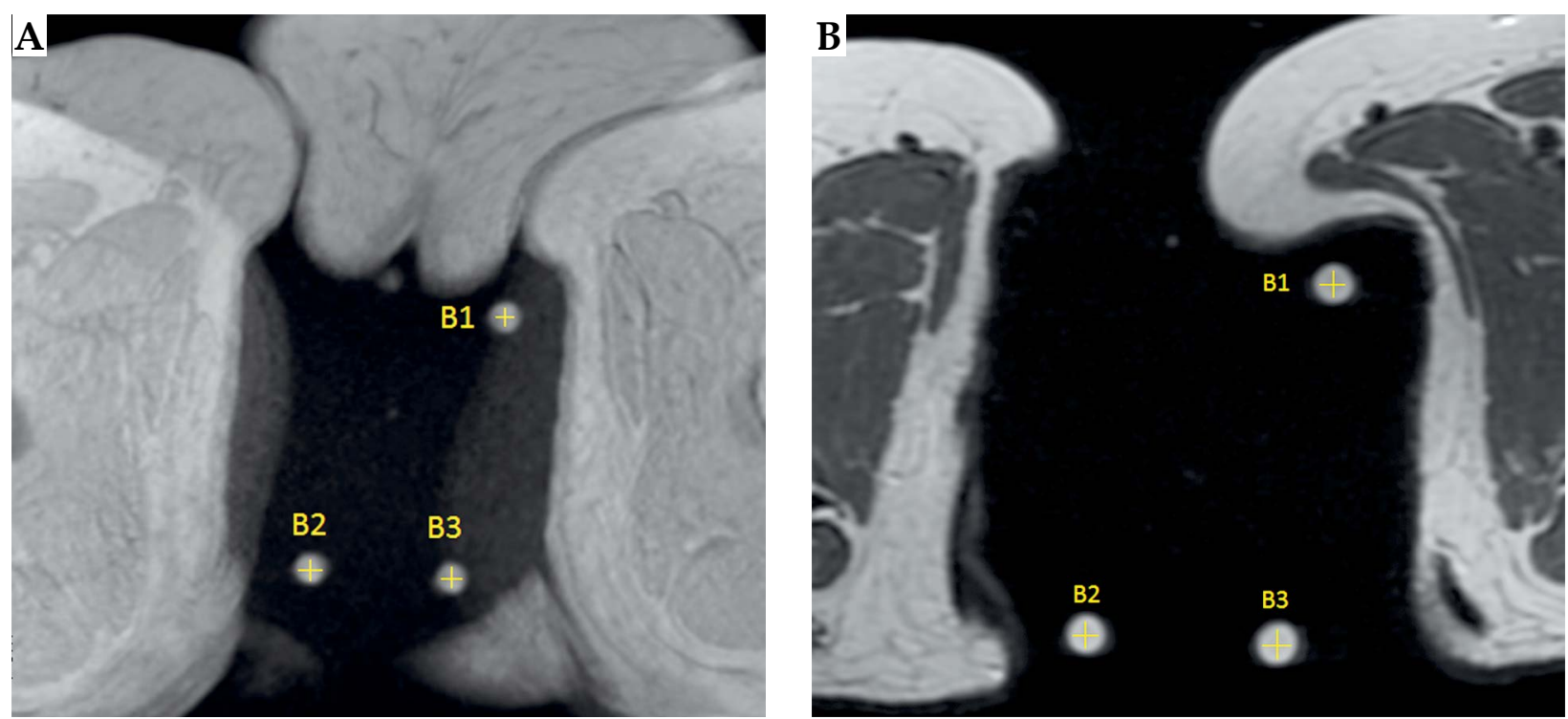

Fig. 2. T1-weighted image (A) and T2-weighted image (B). A-vitamin pellets produce a strong signal in both sequences

\section{Inter-observer analysis}

To evaluate the reconstruction method, an inter-observer study was performed. Two different medical physicists independently reconstructed 3 implants for the first set of patients (3D T1W), with 16, 18, and 16 needles, and 3 implants for the second set of patients (T2W) with 15, 17 , and 16 needles, which result in a total of 50 and 48 needles, respectively. The main source of variability comes from the selection of the points B1, B2, B3 as the centers of the A-vitamin pellets, and the choice of the P1 and P2 points for each needle on the MR images. Differences in the coordinates of the tip of the needles obtained by both observers were measured. Both mean values and standard deviations for each set of patients were reported.

\section{Results}

\section{Applicator reconstruction}

The algorithm have been implemented in a homemade spreadsheet and requires as input data the coordinates of the centers of the A-vitamin pellets (B1, B2, and B3), offset distance $(7 \mathrm{~mm})$, needle length $(200 \mathrm{~mm})$, the free length (measured by the physician during the insertion procedure), and two points (P1, P2) to define the direction of each needle. The output generated for each needle is its tip coordinates and the coordinate of the point defined by the intersection of the straight line defining the needle with the reference plane. At present, these data are introduced in the TPS manually.

During MR acquisition, the procedure is monitored to ensure that there is no patient movement in between the different sequences of images acquired. Figure 3 shows a typical example of needle coordinates determination on two different planes. The selection of the two points required for each needle is done according to the quality of the image. The planes chosen can be different depending on the particular needle analyzed. The points P1 and P2 can be selected anywhere on the needle, as mentioned above. However, in some slices, the small black signal produced by some needle may be difficult to distinguish due to heterogeneities in the surrounding tissue. This is not an issue, since one can easily select any other slice where the visibility is better. Figure 4 shows an example of a reconstructed needle in both $\mathrm{T} 1 \mathrm{~W}$ (left) and $\mathrm{T} 2 \mathrm{~W}$ (right). Visual inspection of the position of the tip relative to the void at the end of the needle is also used as an extra check for detecting errors during the reconstruction process.

\section{Inter-observer analysis}

Inter-observer differences on needles tips coordinates were small. For the patients of group 1, the mean difference was $0.20 \mathrm{~mm}$ with a standard deviation (SD) of $0.02 \mathrm{~mm}$ and a range of $0.03-0.75 \mathrm{~mm}$. In the case of patients of group 2, the mean difference was $0.8 \mathrm{~mm}$ with a SD of $0.1 \mathrm{~mm}$, a range of $0.2-1.1 \mathrm{~mm}$.

Inter-observer variation was larger in the case of reconstruction based on T2 WI. This can be attributed to the slightly larger voxel size in the longitudinal direction. Since differences between observers in the reconstruction process are small (below $1 \mathrm{~mm}$ ), we think that the main source of inaccuracy in the overall reconstruction process is the precise measurement of the free length of the needles during their insertion in the operating room. This is done with a ruler of $1 \mathrm{~mm}$ sensibility.

\section{Discussion}

It may be a matter of discussion if needles are really a straight line. On our experience, we have not reported any case where the needle had been bended during the insertion procedure, so the straight line obtained with two points fits well the void seen on MR images.

During commissioning and using phantom measurements, one can well correlate the actual tip of the needle with the black signal produced at its end on MR images. 
A

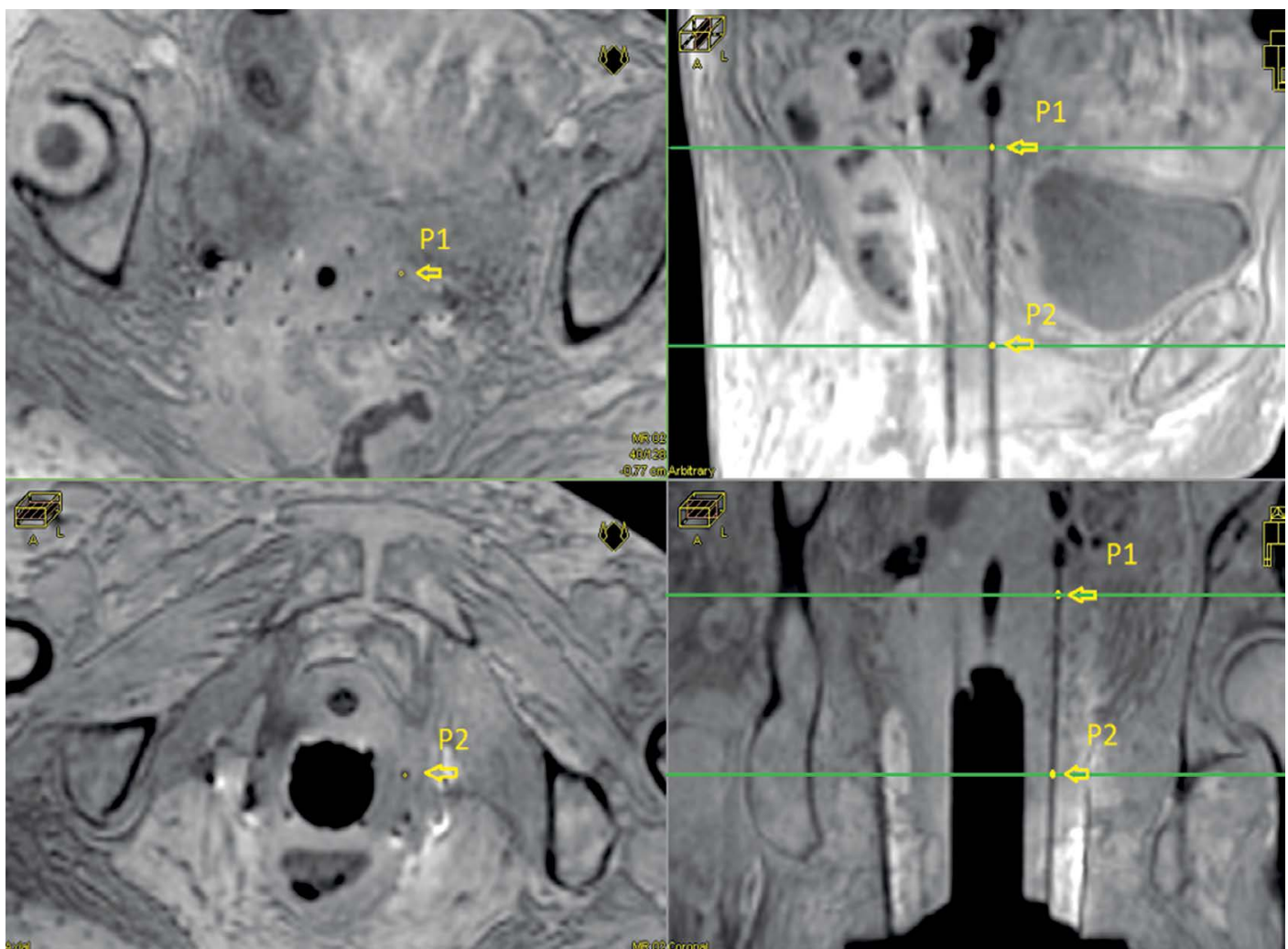

B

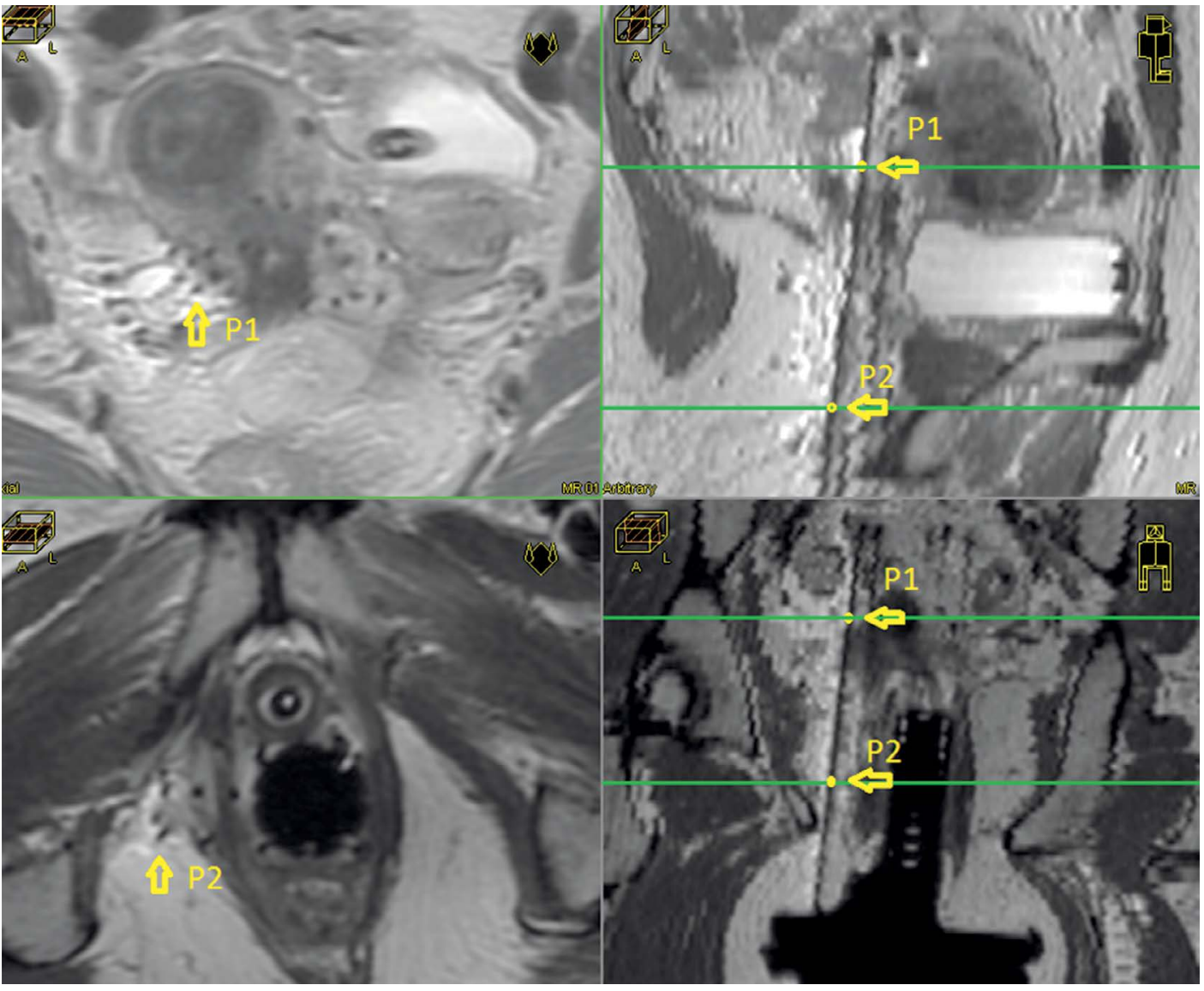

Fig. 3. A) 3D T1W MRI images showing two different paraxial slices (left) and the choice of two points to determine the direction of one needle. Upper right and lower right show the position of points P1 and P2 in parasagittal and para-coronal views, respectively. B) T2 MRI images showing two different paraxial slices (left) and the choice of two points to determine the direction of one needle. Upper right and lower right show the position of points P1 and P2 in para-sagittal and para-coronal views, respectively 

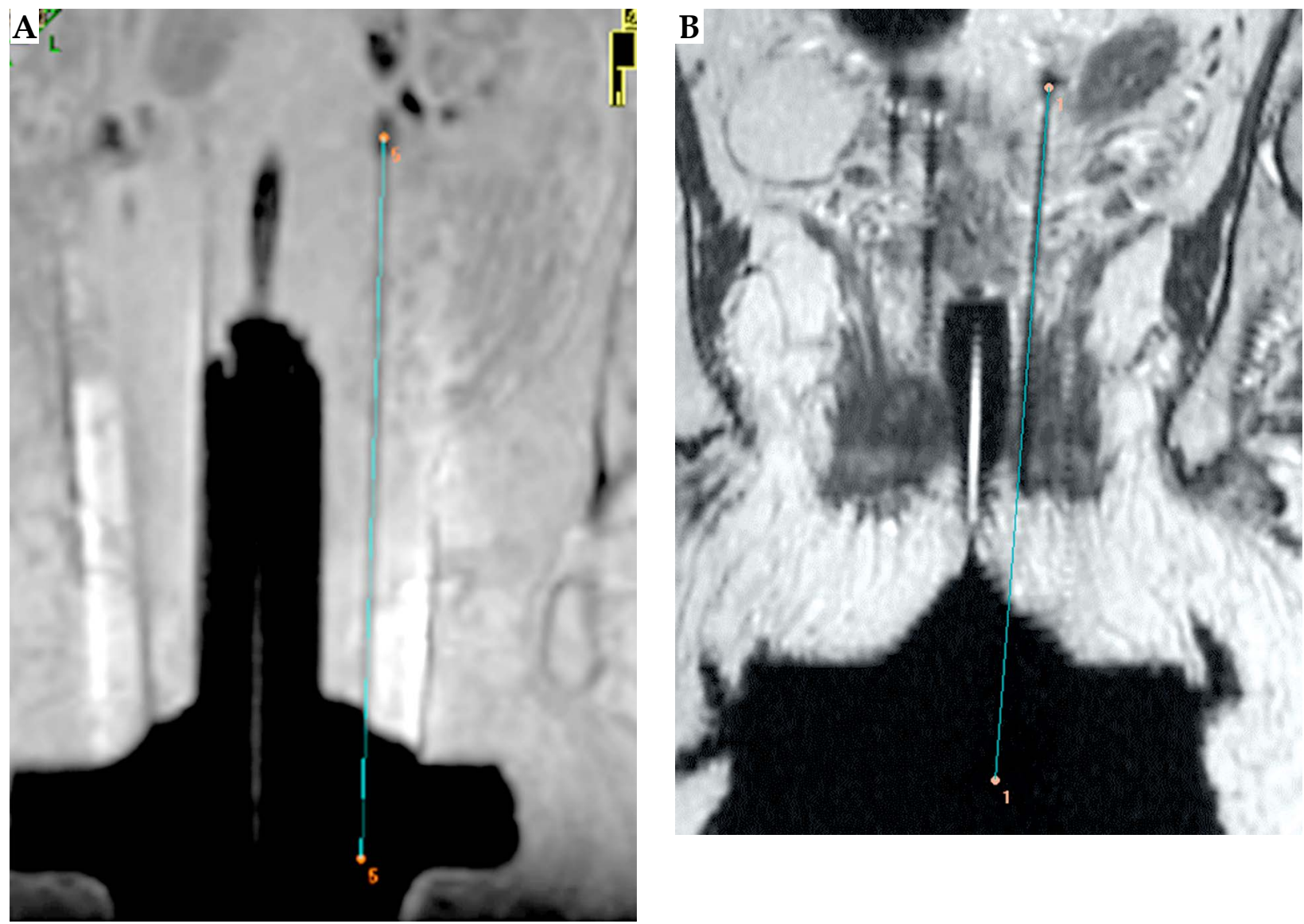

Fig. 4. The needle tip obtained with our method and its position relative to the artifact in T1W (A) and T2W (B)

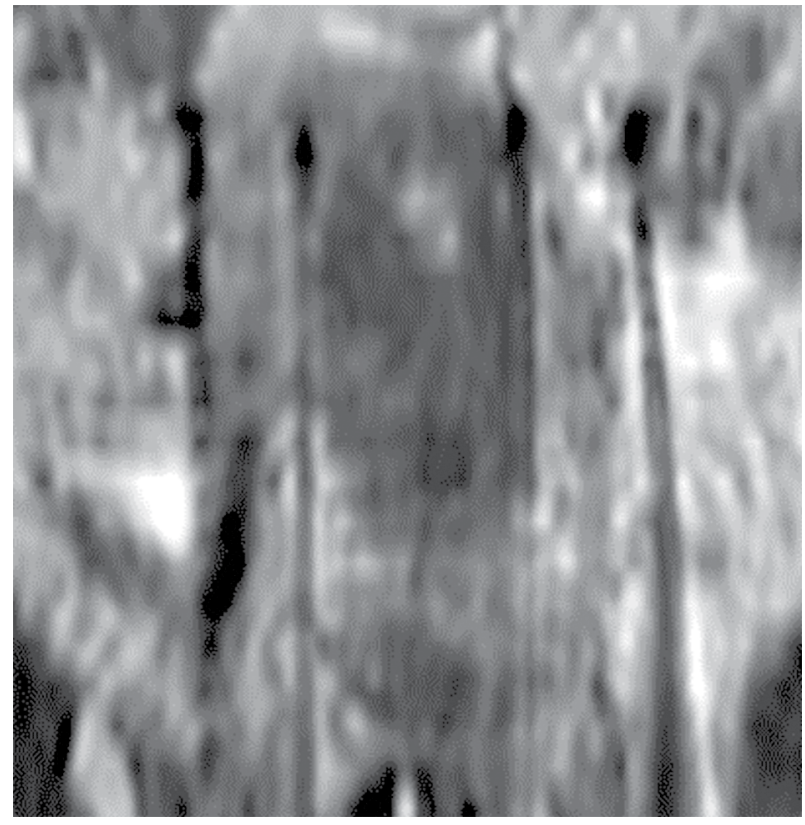

Fig. 5. Example with poor visibility of artifact at the end of the needle

This is done by registering CT and MR scans and finding the position of the needle tip according to its position found in the CT scan. The procedure has been previous- ly described in the work by Haack et al. [13]. Titanium needles produce a clear and reproducible artefact pattern. They generate bright regions in both sides of the needles in a coronal view that can be used to locate the tip position. Nevertheless, unlike phantoms, patient tissues surrounding the needles present heterogeneities that complicate the accurate identification of these artifact patterns, see Figure 5. By using the method presented in this paper, this is not an issue, since it does not depend on the visibility of the artefact at the end of every needle.

Applicator reconstruction for MRI based BT can be performed in the same sequence used for contouring and planning (mainly T2 WI) but some considerations have to be taken into account. The first one is whether reconstruction is based on library plans, where the geometry of the applicator is previously implemented in the TPS, or done directly from MR images. The former method applies well in the case of rigid applicators. In the second option, a spacing of $4 \mathrm{~mm}$ may be too large for reconstruction purposes since the sagittal or coronal reconstructed views presents sub-optimal resolution. To overcome this problem, a sagittal acquisition with small spacing may be performed. Nevertheless, in cases we focused in this manuscript, that is patients with large diseases, implants are large and a great number of images may be required to cover the extension of the whole implant. This may increase significantly the acquisition time and therefore make small spacing sagittal acquisition unpractical. In an 
attempt to overcome this limitation, two different paths may be followed. The first one will be to choose a 3D T1 sequence for reconstruction with good resolution in all directions, while a T2 WI sequence is used for contouring. A different possibility will be to use instead a $2 \mathrm{~mm}$ axial T2 WI sequence that, after being validated by the physician, will be used for contouring. Using any of them, typical acquisition times ranges between 7-10 min.

Many authors have evaluated different applicators reconstruction methods based on MR images. Haack et al. [13] included on their MR applicator reconstruction study the titanium needles. The authors concluded that the reconstruction could be performed by considering the needle alone together with its artefact on the MR images and evaluating the tip as the center of the void artefact. As shown in Figure 4, our procedure gives automatically the tip location within $1 \mathrm{~mm}$ margin. The tips are located in the center of the "black" signal. This reinforces our confidence on the course of action proposed in this manuscript.

Schindel et al. [19] studied different MRI interstitial/ intracavitary markers; although no titanium needles were tested. They were not considered because dummy-marker catheters cannot be visualized inside the source guide tubes due to the appearance of susceptibility artefacts.

The work of Petit et al. [18] describes a reconstruction method based on a $1.5 \mathrm{~T}$ MR for the case of a titanium applicator. The Rotterdam applicator, in which the titanium tubes are $3 \mathrm{~mm}$ in diameter, was chosen in this study. Once assembled, the Rotterdam applicator may be considered as rigid, so it can be reconstructed with good accuracy by using a library previously implemented on the TPS

The commercial version of the TPS applicator library available in our hospital does not include pure interstitial applicator such as the standard MUPIT. In this case, in a work in progress, the template can be implemented into the TPS applicator library, although each individual case has to be modelled independently since each patient requires his own needle arrangement. Once this is done, the reconstruction can be performed with the aid of the anchor points (A-vitamin pellets) defined previously in the library. Nevertheless, the whole applicator cannot be considered completely rigid. In the perineal plates edges it is rigid but as the needles go deeper into the patient they may deviate slightly from its modelled position. This is caused for the looseness in the holes during the drilling process of the plates, and small adjustments need to be done when the TPS library is used for reconstruction. We are currently working on further studies along this line.

\section{Conclusions}

We have developed a new method for titanium needle direct reconstruction in interstitial implants based exclusively on MR images. It can be applied either to T1- or T2-weighted sequences allowing the use of only one sequence (T2W) either for contouring or reconstruction purposes. The proposed algorithm is robust, since it is independent of the visibility of the artefact at the end of the needle, and not prone to inter-observer variation.
Furthermore, it can be implemented easily on a spreadsheet. The A-vitamin pellets can be used as anchor points when implement the applicator into the library plan of the TPS. Therefore, the algorithm presented is simple and easy to implement into clinical practice.

\section{Acknowledgments}

The current study was supported in part by Generalitat Valenciana under Project PROMETEOII/2013/010 and by Spanish Government under Project No. FIS201342156. This study was partially supported by Lorca Marin S.A. Murcia (Spain).

\section{Disclosure}

Authors report no conflict of interest.

\section{References}

1. Dimopoulos JC, Schard G, Berger D et al. Systematic evaluation of MRI findings in different stages of treatment of cervical cancer: potential of MRI on delineation of target, pathoanatomic structures, and organs at risk. Int J Radiat Oncol Biol Phys 2006; 64: 1380-1388.

2. Viswanathan AN, Dimopoulos J, Kirisits C et al. Computed tomography versus magnetic resonance imaging based contouring in cervical cancer brachytherapy: results of a prospective trial and preliminary guidelines for standardized contours. Int J Radiat Oncol Biol Phys 2007; 68: 491-498.

3. Viswanathan A, Thomadsen B. American Brachytherapy Society consensus guidelines for locally advanced carcinoma of the cervix. Part I: general principles. Brachytherapy 2012; 11: 33-46.

4. Haie-Meder C, Pötter R, Van Limbergen E et al. Recommendations from Gynecological (GYN) GEC-ESTRO Working Group (I): concepts and terms in 3D image based 3D treatment planning in cervix cancer brachytherapy with emphasis on MRI assessment of GTV and CTV. Radiother Oncol 2005; 74: 235-245.

5. Pötter R., Haie-Meder C, Van Limbergen E et al. Recommendations from gynaecological (GYN) GEC ESTRO working group (II): concepts and terms in 3D image-based treatment planning in cervix cancer brachytherapy-3D dose volume parameters and aspects of 3D image-based anatomy, radiation physics, radiobiology. Radiother Oncol 2006; 78: 67-77.

6. Kiristis C, Lang S, Dimopoulus J et al. The Vienna applicator for combined intracavitary and interstitial brachytherapy of cervical cancer: design, application, treatment planning and dosimetric results. Int J Radiat Oncol Biol Phys 2006; 65: 624-630.

7. Nomden CN, de Leeuw AA, Moerland MA et al. Clinical use of the Utrech applicator for combined intracavitary/interstitial brachytherapy treatment in locally advanced cervical cancer. Int J Radiat Oncol Biol Phys 2012; 82: 1424-1430.

8. Martinez A, Cox RS, Edmundson GK. A multiple-site perineal applicator (MUPIT) for treatment of prostatic, anorectal and gynecologic malignancies. Int J Radiat Oncol Biol Phys 1984; 10: 297-305.

9. Syed A, Puthawala AA, Neblett D et al. Transperineal interstitial intracavitary "syed-neblett" applicator in the treatment of carcinoma of the uterine cervix. Endocuriether Hypertherm 1986; 2: 1-13.

10. Nag S, Erickson B, Thomadsen B et al. The American Brachytherapy society recommendations for high dose rate brachytherapy for carcinoma of the cervix. Int J Radiat Oncol Biol Phys 2000; 48: 201-211. 
11. Viswanathan A, Erickson B, Rownd J. Image guided approaches to interstitial brachytherapy. In: Gynecologic radiation therapy: novel approaches to image-guidance and management. Viswanathan A, Kiristis C, Erickson B, Pötter R (eds.). Springer, Berlin-Heidelberg 2011; 247-259.

12. Rodriguez S, Richart J, Otal A et al. A new template for MRIbased intracavitary/interstitial gynecologic brachytherapy: design and clinical implementation. J Contemp Brachytherapy 2015; 7: 265-272.

13. Haack S, Nielsen SK, Lindegaard JC et al. Applicator reconstruction in MRI 3D image-based dose planning of brachytherapy for cervical cancer. Radiother Oncol 2009; 91: 187193.

14. Perez-Calatayud J, Kuipers F, Ballester F et al. Exclusive MRI-based tandem and colpostats reconstruction in gynaecological brachytherapy treatment planning. Radiother Oncol 2009; 91: 181-186.

15. Chargari C, Magné N, Dumas I et al. Physics contribution and clinical outcome with 3D-MRI-based pulsed-dose-rate intracavitary brachytherapy in cervical cancer patients. Int $J$ Radiat Oncol Biol Phys 2009; 74: 133-139.

16. Hellebust TP, Kirisits C, Berger D et al. Recommendations from Gynaecological (GYN) GEC-ESTRO Working Group: Considerations and pitfalls in commissioning and applicator reconstruction in 3D image-based treatment planning of cervix cancer brachytherapy. Radiother Oncol 2010; 96: 153-160.

17. Tanderup K, Hellebust TP, Lang S et al. Consequences of random and systematic reconstruction uncertainties in 3D image based brachytherapy in cervical cancer. Radiother Oncol 2008; 89: 156-163.

18. Petit S, Wielopolski P, Rijnsdorp R et al. MR guided applicator reconstruction for brachytherapy cervical cancer using the novel titanium Rotterdam applicator. Radiother Oncol 2013; 100: 88-92.

19. Schindel J, Muruganandham M, Pigge C et al. Magnetic resonance imaging (MRI) markers for MRI-guided high-doserate brachytherapy: novel marker-flange for cervical cancer and marker catheters for prostate cancer. Int J Radiat Oncol Biol Phys 2013; 86: 387-393. 